\title{
Necrotizing Keratitis Caused by Acyclovir-Resistant Herpes Simplex Virus
}

\author{
Koji Toriyama Tomoyuki Inoue Takashi Suzuki Takeshi Kobayashi \\ Yuichi Ohashi \\ Department of Ophthalmology, Ehime University School of Medicine, Toon, Japan
}

\section{Key Words}

Herpes simplex virus - Acyclovir-resistant herpes simplex virus · Necrotizing keratitis · Fungal infection $\cdot$ Real-time polymerase chain reaction

\begin{abstract}
Background: We report a case of necrotizing keratitis caused by acyclovir (ACV)-resistant herpes simplex virus (HSV) with a clinical appearance similar to a previous fungal keratitis infection. Methods: Observational case report. Results: Penetrating keratoplasty was performed in the left eye with a history of herpetic keratitis that resolved with periodic treatment with ACV ointment and a topical steroid. The left eye was painful and red with an abscess and corneal erosion in the peripheral donor cornea. Examination of the scraped corneal epithelium by light microscopy and culturing identified Candida albicans; polymerase chain reaction (PCR) was negative for human herpes viruses. After antifungal treatment, the ocular pain gradually decreased and the lesions slowly improved but recurred with a similar clinical appearance. A second light microscopy examination and cultures were negative for pathogens including C. albicans. PCR was positive for HSV-1 DNA; treatment with 3\% topical ACV ointment was unsuccessful. A third examination showed only HSV-1 DNA. Despite antiviral ACV ointment, no clinical improvement occurred based on the HSV DNA copy numbers, which were the same before and after treatment, indicating a possible ACVresistant strain. When topical trifluorothymidine was substituted for ACV, clinical improvement occurred and the HSV DNA copy numbers decreased. Conclusion: Necrotizing keratitis induced by ACV-resistant HSV occurred independently after fungal keratitis, with a similar clinical appearance in this case, making diagnosis and treatment difficult. Monitoring the HSV DNA load by real-time PCR could be useful for refractory cases even with atypical clinical appearances.


Toriyama et al.: Necrotizing Keratitis Caused by Acyclovir-Resistant Herpes Simplex Virus

\section{Introduction}

Some cases of herpes simplex virus (HSV) keratitis refractory to topical acyclovir (ACV) treatment were previously reported as epithelial keratitis [1,2]. Conventional diagnosis of an ACV-resistant HSV keratitis using a viral culture following in vitro drug sensitivity testing [1] is generally difficult and complicated. Recently, we reported a simple and useful method for diagnosing drug-resistant herpetic infection using real-time polymerase chain reaction (PCR) by monitoring the changes in the viral DNA copy numbers before and after ACV treatment [3]. We describe a case of ACV-resistant HSV keratitis with an atypical extensive necrotizing focus diagnosed using our proposed estimation method.

\section{Case Report}

A 54-year-old man with a clinical diagnosis of herpetic keratitis had undergone penetrating keratoplasty in the left eye 6 years previously for recurrent keratouveitis, which resolved after periodic treatment with ACV ointment and a topical steroid. The patient had ocular pain and redness in his left eye and periodically received topical antibiotics and a steroid for 1 month; however, the symptoms gradually worsened despite treatment, and he was referred to our clinic. The best-corrected visual acuity in the affected eye was 20/2,000 and the intraocular pressure was $10 \mathrm{~mm} \mathrm{Hg}$. Slit-lamp examination showed a small abscess with corneal erosion in the peripheral donor cornea that manifested as a suture abscess with corneal infiltrates, mild ciliary injection, and no anterior chamber inflammation (day 0; fig. 1a). The sensitivity of the left cornea decreased to $20 \mathrm{~mm}$ compared with $60 \mathrm{~mm}$ in the right eye. The right eye was normal. The suture at the focus was removed and wide corneal scraping was performed for cytopathologic examination and culturing to detect pathogenic microorganisms such as bacteria, fungus, or Acanthamoeba. Real-time PCR analysis was performed to detect human herpes viruses (HHVs; HSV type 1 or 2, varicella zoster virus, Epstein-Barr virus, cytomegalovirus, and HHV-6, -7, and -8) [4, 5]. Only Candida albicans was identified by culturing.

The patient was diagnosed with fungal keratitis and treated with topical $0.2 \%$ fluconazole hourly and 1.5\% topical levofloxacin 3 times/day for 2 weeks. The ocular pain gradually decreased and the lesions slowly improved (day 14; fig. 1b). However, the lesion recurred and increased, showing a similar clinical appearance despite the same antifungal treatment (day 21; fig. 1c). Because of the unresponsiveness to the antifungal agent, a second corneal scraping was obtained to repeat the same microbiologic examinations. Light microscopy and the cultures were negative for pathogens including C. albicans. The PCR results were positive only for HSV-1 DNA (3.4 × $10^{4}$ copies/sample). Based on these positive PCR results for HSV1 DNA and the past clinical background, the patient was suspected of having HSV keratitis and was treated with 3\% topical ACV ointment 5 times/day and $0.5 \%$ topical levofloxacin 3 times/day for 2 weeks. However, the necrotizing lesions did not improve (days 28, 35; fig. $1 \mathrm{~d}$, e, respectively). In the third scraping, only HSV-1 DNA (5.7 $\times 10^{4}$ copies/sample) was detected.

$\mathrm{ACV}$ as a specific anti-HSV agent generally can improve the herpetic keratitis along with a substantial decrease in the HSV DNA copy numbers compared with before treatment; however, the HSV viral load in the current case did not decrease as a result of ACV treatment, and the only detected pathogen was HSV, indicating that the HSV detected in these lesions might be ACV resistant. Although this necrotizing focus was atypical of the clinical appearance of ACV-resistant HSV keratitis reported previously [1, 2], we suspected that this focus 
Toriyama et al.: Necrotizing Keratitis Caused by Acyclovir-Resistant Herpes Simplex Virus

was induced by ACV-resistant HSV based on this diagnostic concept in addition to negative results for other pathogens including C. albicans. We substituted topical 1\% trifluorothymidine (TFT) solution, which was reported to be effective for ACV-resistant HSV keratitis [1, 2]. The ocular pain gradually decreased, and the lesions slowly improved. Real-time PCR of the epithelial scraping obtained 2 weeks after the start of TFT therapy did not detect HSV-1 DNA (day 49; fig. 1f), and by day 70, the keratitis had healed with corneal scarring (fig. 1g). There has been no recurrence at 1 year.

\section{Discussion}

Generally, ACV-resistant HSV has a low virulence because of the loss or change in enzyme activity of the viral thymidine kinase $[1,2]$. To the best of our knowledge, this is the first case report of ACV-resistant HSV keratitis with an extensive necrotizing focus diagnosed using our proposed monitoring of the changes in the viral DNA copy numbers by real-time PCR before and after ACV treatment. The mechanism of necrotizing keratitis is thought to be due to retained viral antigen within the corneal stroma. This antigen triggers an antigenantibody-complement cascade that induces polymorphonuclear neutrophil infiltration, leading to destructive inflammation [6]. In the current case, the reason for severe inflammation caused by ACV-resistant HSV was unclear, but one possibility is that the preceding fungal infection might have boosted the local immunologic status and activated polymorphonuclear neutrophils on the graft, and that condition induced extensive necrotizing focus.

During the clinical course, the current patient did not have mixed infections of Candida and HSV because the case was positive for Candida and negative for HSV based on the first microbiologic examination and negative for Candida and positive for HSV based on the second examination. ACV-resistant HSV keratitis occurred independently after fungal keratitis. The pathogens changed from Candida to HSV with a similar clinical appearance, which can complicate the disease status and make diagnosis and treatment more difficult.

Although the clinical background in this case was consistent with the characteristics of ACV-resistant keratitis described previously in the details of the therapeutic history, steroid usage, and refractoriness to ACV therapy and/or a post-penetrating keratoplasty eye [1, 2], accurate diagnosis of ACV-resistant HSV keratitis is difficult especially with an atypical clinical appearance; the variations in the clinical findings of ACV-resistant HSV remain to be elucidated. Monitoring the changes in the viral DNA load by real-time PCR permits accurate diagnosis of ACV-resistant herpetic keratitis even in a case with an atypical clinical appearance.

\section{Disclosure Statement}

The authors have no proprietary interest in any aspect of this work.

\section{References}

1 Yao YF, Inoue Y, Kase T, Uchihori Y, Mori Y, Ohashi Y: Clinical characteristics of acyclovir-resistant herpetic keratitis and experimental studies of isolates. Graefes Arch Clin Exp Ophthalmol 1996;234:S126-S132.

2 Zhang W, Suzuki T, Shiraishi A, Shimamura I, Inoue Y, Ohashi Y: Dendritic keratitis caused by an acyclovirresistant herpes simplex virus with frameshift mutation. Cornea 2007;26:105-106. 
Toriyama et al.: Necrotizing Keratitis Caused by Acyclovir-Resistant Herpes Simplex Virus

3 Inoue T, Kawashima R, Suzuki T, Ohashi Y: Real-time polymerase chain reaction for diagnosing acyclovirresistant herpetic keratitis based on changes in viral DNA copy number before and after treatment. Arch Ophthalmol 2012;130:1462-1464.

4 Inoue T, Kandori M, Takamatsu F, Hori Y, Maeda N: Corneal endotheliitis with quantitative polymerase chain reaction positive for human herpesvirus 7. Arch Ophthalmol 2010;128:502-503.

5 Inoue T, Takamatsu F, Kubota A, Hori Y, Maeda N, Nishida K: Human herpesvirus 8 in corneal endotheliitis resulting in graft failure after penetrating keratoplasty refractory to allograft rejection therapy. Arch Ophthalmol 2011;129:1629-1630.

6 Meyers-Elliott RH, Chitjian PA: Immunopathogenesis of corneal inflammation in herpes simplex virus stromal keratitis: role of the polymorphonuclear leukocyte. Invest Ophthalmol Vis Sci 1981;20:784-798.
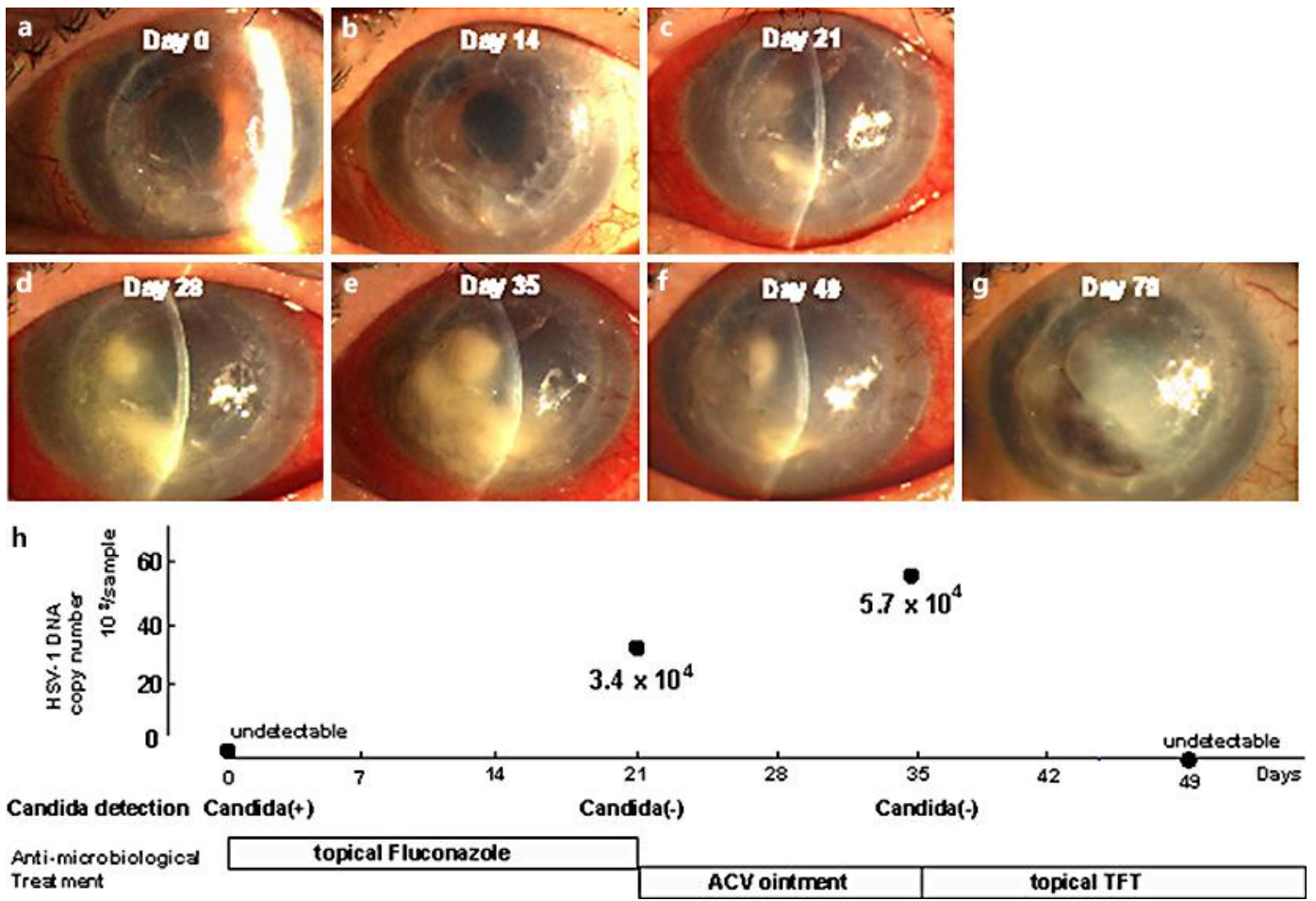

Fig. 1. Slit-lamp photographs of the therapeutic outcomes with antimicrobiologic therapy. a A small abscess with corneal erosion in the peripheral donor cornea induced by $C$. albicans with negative results for HSV on real-time PCR (day 0). b Resolution of the lesions after antifungal treatment (day 14). c A recurrent lesion despite the same antifungal treatment (day 21). d, e Progressive necrotizing lesions despite ACV treatment (days 28, 35, respectively). f Gradual lesion improvement after topical TFT therapy (day 49). $\mathbf{g}$ Resolution of the lesions (day 70). $\mathbf{h}$ The relationship between the therapeutic outcomes with antifungal therapy, ACV, and TFT antiviral treatment and microbiologic results of Candida detection and HSV DNA copy numbers. 